\title{
Is It Time to Add Tumor Regression Grade into the ypN Category?
}

\author{
Yin-Kai Chao, MD \\ Division of Thoracic Surgery, College of Medicine, Chang Gung Memorial Hospital-Linkou, Chang Gung University, \\ Taoyuan, Taiwan
}

Esophageal cancer is characterized by aggressive biological behavior and generally portends an unfavorable clinical course and outcomes. ${ }^{1}$ Nodal metastases are commonly recognized as the main predictor of disease recurrence. ${ }^{2}$ In addition to the number of metastatic nodes, interest in identifying other lymph-node-related pathological characteristics that could further refine prognostic stratification is mounting. These variables include the anatomic location of nodal metastases, the presence of extra- or intracapsular spread, and the lymph node ratio. ${ }^{3-5}$ While the available evidence is largely derived from patients who had undergone primary esophagectomy, candidates for upfront surgery are less frequently seen in current surgical practice. ${ }^{3-5}$

The use of neoadjuvant chemotherapy or chemoradiotherapy for patients with esophageal cancer has increased dramatically following the publication of the CROSS trial in $2012 .{ }^{6}$ On analyzing survival figures, the results of this landmark study indicated that patients with regionally advanced disease who received neoadjuvant therapy had a clear survival advantage over those who were treated with primary esophagectomy alone, especially in presence of esophageal squamous cell carcinoma (ESCC). ${ }^{6,7}$ However, the amount of pathological information that can be extracted from surgical specimens has been significantly affected by the introduction of neoadjuvant therapy. Specifically, the extent of tumor response to neoadjuvant

(C) Society of Surgical Oncology 2021

First Received: 8 November 2020

Accepted: 18 November 2020;

Published Online: 3 January 2021

Y.-K. Chao, MD

e-mail: chaoyk@cgmh.org.tw therapy-as expressed by the tumor regression grade (TRG) of the primary tumor (TRG-tumor) and/or metastatic lymph nodes (TRG-LN)—came into play. ${ }^{8-10}$ While it is generally accepted that a more pronounced TRG-tumor after neoadjuvant therapy portends favorable outcomes, the prognostic significance of TRG-LN is a matter of ongoing debate. ${ }^{11,12}$ In this issue of the Annals of Surgical Oncology, Hsu et al. investigate whether TRG-LN may predict survival outcomes in patients with ESCC who had been treated with neoadjuvant chemoradiotherapy followed by surgery. ${ }^{13}$ In their study, TRG-LN was scored as follows: 0 , negative nodes without evidence of tumor involvement or regression [cNO $\rightarrow$ ypNO]; 1, complete regression of nodal involvement [ $\mathrm{cN}+\rightarrow \mathrm{ypN} 0]$; 2 , partial regression of nodal involvement with $<50 \%$ of viable tumor; and 3 , no significant regression of nodal involvement with $\geq 50 \%$ of viable tumor. The results showed favorable overall and disease-free survival figures for patients with TRG-LN scores of $0-1$ (without significant intergroup differences). Conversely, poor survival outcomes were observed for those with scores of $2-3$, again without intergroup differences. On multivariate analysis, TRG-LN scores of 2-3 were retained in the model as the only independent adverse predictor of OS. Thus, TRG-LN was found to outweigh the prognostic value of TRG-tumor, ypT stage, and the R0 category. Nevertheless, there are numerous factors that could have had an effect on study findings that could mitigate the prognostic value of TRG-LN for survival outcomes.

First, once there was evidence of persistent nodal involvement after neoadjuvant therapy, it is surprising that no survival differences were observed for patients with $<50 \%$ versus $\geq 50 \%$ of viable tumor (i.e., score 2 vs. score 3 ). Because 5 -year overall survival rates were equally dismal (only 8\%!) regardless of regression extent, the use 
of TRG-LN would add limited prognostic information over the current ypN stage category. In this scenario, the authors should be encouraged to conduct additional subgroup analyses in patients with minimal residual nodal disease (i.e., $<10 \%$ of viable tumor) to investigate whether their survival figures are similar to those observed in patients with complete regression of nodal involvement. ${ }^{14}$ Second, the lack of survival differences in patients with TRG-LN scores of $0-1$ does not imply that TRG-LN is without prognostic value in the ypNO group. In this regard, the adverse significance of tumor-regressed nodal numbers for survival outcomes has been repeatedly reported-possibly as the result of a significant tumor burden. ${ }^{15,16}$ However, it is not possible to determine from the study by Hsu et al. how many nodes had signs of preexisting tumor involvement. Third, and even more importantly, a key prerequisite for accurate TRG-LN assessment is the systematic evaluation of the entire nodal structure. Theoretically, an en bloc lymph node resection (i.e., complete removal of lymph nodes with the surrounding adipose tissue as a lump following anatomical landmarks surrounding the nodes) would be ideal to achieve this goal. Because mediastinal nodes and their surrounding sheaths are more fragile and prone to tear upon dissection compared with abdominal nodes, significant variations in the thoroughness of their assessment across different institutions, surgeons, and pathologists can be expected. Undoubtedly, the presence of fragmented nodes may lead to erroneous TRG-LN estimations. Although the study by Hsu et al. does not provide information on their handling and does not mention a potential interobserver bias among different pathologists, the authors should be commended for their attempt to address an interesting research question for patients with ESCC who receive neoadjuvant therapy. The incorporation of TRG-LN assessment into the current staging system for esophageal cancer will ultimately depend on the results of large multicenter international research efforts. Similarly, the prognostic significance of TRG-LN in esophageal malignancies is to be confirmed by following a larger cohort for a longer period of time.

DISCLOSURE The author declares no conflict of interest in relation to this manuscript.

\section{REFERENCES}

1. Enzinger PC, Mayer RJ. Esophageal cancer. $N$ Engl J Med. 2003;349(23):2241-52.

2. Kayani B, Zacharakis E, Ahmed K, Hanna G. Lymph node metastases and prognosis in oesophageal carcinoma-a systematic review. Eur J Surg Oncol. (EJSO). 2011;37(9):747-53.
3. Tachimori Y, Ozawa S, Numasaki H, et al. Efficacy of lymph node dissection for each station based on esophageal tumor location. Esophagus. 2016;13(2):138-45.

4. Udagawa $\mathrm{H}$, Ueno $\mathrm{M}$, Shinohara $\mathrm{H}$, et al. The importance of grouping of lymph node stations and rationale of three-field lymphoadenectomy for thoracic esophageal cancer. J Surg Oncol. 2012;106(6):742-7.

5. Tan Z, Ma G, Yang H, Zhang L, Rong T, Lin P. Can lymph node ratio replace pn categories in the tumor-node-metastasis classification system for esophageal cancer? $J$ Thorac Oncol. 2014;9(8):1214-21.

6. van Hagen P, Hulshof M, Van Lanschot J, et al. Preoperative chemoradiotherapy for esophageal or junctional cancer. $N$ Engl $J$ Med. 2012;366(22):2074-84.

7. Shapiro J, van Lanschot JJB, Hulshof MC, et al. Neoadjuvant chemoradiotherapy plus surgery versus surgery alone for oesophageal or junctional cancer (CROSS): long-term results of a randomised controlled trial. Lancet Oncol. 2015;16(9):1090-8.

8. Chao Y-K, Chang C-B, Chuang W-Y, et al. Correlation between tumor regression grade and clinicopathological parameters in patients with squamous cell carcinoma of the esophagus who received neoadjuvant chemoradiotherapy. Medicine. 2015;94(34):e1407.

9. Puetz K, Bollschweiler E, Semrau R, Mönig SP, Hölscher AH, Drebber U. Neoadjuvant chemoradiation for patients with advanced esophageal cancer-Which response grading system best impacts prognostic discrimination? Histopathology. 2019;74(5):731-43.

10. Barbour AP, Jones M, Gonen M, et al. Refining esophageal cancer staging after neoadjuvant therapy: importance of treatment response. Ann Surg Oncol. 2008;15(10):2894-902.

11. Urakawa S, Makino T, Yamasaki M, et al. Lymph node response to neoadjuvant chemotherapy as an independent prognostic factor in metastatic esophageal cancer. Ann Surg. 2019. https://doi.org/ 10.1097/sla.0000000000003445.

12. Langer $\mathrm{R}$, Becker $\mathrm{K}$, Zlobec $\mathrm{I}$, et al. A multifactorial histopathologic score for the prediction of prognosis of resected esophageal adenocarcinomas after neoadjuvant chemotherapy. Ann Surg Oncol. 2014;21(3):915-21.

13. Hsu P-K, Yeh Y-C, Chien L-I, Huang C-S, Hsu H-S. Clinicopathological significance of pathologic complete lymph node regression after neoadjuvant chemoradiotherapy in esophageal squamous cell carcinoma. Ann Surg Oncol. 2020. https://doi.org/ 10.1245/s10434-020-09363-z.

14. Chao YK, Chuang WY, Chang HK, Tseng CK, Yeh CJ, Liu YH. Prognosis of patients with esophageal squamous cell carcinoma who achieve major histopathological response after neoadjuvant chemoradiotherapy. Eur J Surg Oncol. 2017;43(1):234-9.

15. Philippron A, Bollschweiler E, Kunikata A, et al. Prognostic relevance of lymph node regression after neoadjuvant chemoradiation for esophageal cancer. Paper presented at: Seminars in Thoracic and Cardiovascular Surgery; 2016.

16. Nieman DR, Peyre CG, Watson TJ, et al. Neoadjuvant treatment response in negative nodes is an important prognosticator after esophagectomy. Ann Thorac Surg. 2015;99(1):277-83.

Publisher's Note Springer Nature remains neutral with regard to jurisdictional claims in published maps and institutional affiliations. 